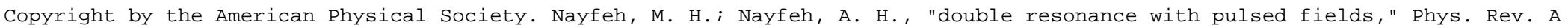
19, 1666 (1979); DOI: http://dx.doi.org/10.1103/PhysRevA.19.1666

PH Y S I C A L REVIEW A

VOL UME 19, NUMBER 4

A P R IL 1979

\title{
Double resonance with pulsed fields
}

\author{
Munir H. Nayfeh \\ Department of Physics, University of Illinois at Urbana-Champaign, Urbana, Illinois 61801
}

A. H. Nayfeh

Virginia Polytechnic Institute and State University, Blacksburg, Virginia 24061

(Received 15 August 1978; revised manuscript received 22 December 1978)

\begin{abstract}
An analytical semiclassical expression is derived for the two-photon transition in a three-level system under the influence of two pulsed fields. We study the Autler-Townes doublet as a function of the time dependence of the field amplitudes and the relaxation widths of the levels. The time variations of the field amplitudes cause shifts in the doublet and modify the radiative decay widths of the levels. The validity of the treatment extends into the intermediate-intensity regime.
\end{abstract}

\section{INTRODUCTION}

Absorption and fluorescence spectra of atoms are modified when the exciting electromagnetic field amplitude rises. Near resonance the fluorescence spectrum consists of three peaks. ${ }^{1}$ The spectrum has been completely calculated based on a classical description of the field ${ }^{2}$ which has since been confirmed by a number of quantum-mechani cal treatments. ${ }^{3}$ The effect of the finite bandwidth of the light source on the properties of fluorescent light has also been investigated, ${ }^{4,5}$ and nonmonochromatic excitation was found to cause a feature in the fluorescence spectrum which is absent under monochromatic excitation. This new feature is an asymmetry in the spectral density when the finite bandwidth excitation is off resonance. ${ }^{5}$ This asymmetry, however, is absent when the excitation is on resonance. These effects may have contributed to recent resonance fluorescence measurements. ${ }^{1,5}$

On the other hand, the absorption spectrum of a second weak light beam probing a transition which has a common level with the saturated transition is predicted to consist of two peaks. This doublet structure was first observed in the microwave region (the Autler-Townes effect). ${ }^{6}$ The optical analog of the Autler-Townes effect has recently received much experimental attention, ${ }^{7,8}$ but although it has been observed in double optical resonance, detailed comparison with theory is very difficult owing to complications in the atomic-level structure. However, a recent experiment, in which a true three-level system was attained by using an atomic beam and optical pumping methods, allowed easy and straightforward comparison with the theory. ${ }^{8}$

Theoretical aspects of double resonance have been treated by many researchers, ${ }^{9}$ but the inter action of two nearly saturating $\mathrm{cw}$ light beams with a three-level system in a stepwise excitation con- figuration has only recently been studied in detail. ${ }^{10,11}$ A numerical treatment of the equation derived from the quantum electrodynamic equations of motion has been given, ${ }^{10}$ and a dressedatom approach for the interaction of an intense laser beam with multilevel atoms has been developed and used to derive analytical expressions for the positions, widths, and weights of the various components of fluorescence and absorption spectra. ${ }^{11}$ In the first step of the dressed-atom approach to resonance fluorescence, one neglects spontaneous emission and determines the energy levels of the combined isolated-atom and laser photons for the dressed atom. Resonance fluorescence can then be treated as spontaneous emission from the dressed-atom system, and double resonance in the stepwise configuration can be treated as absorption of a probe beam by the same system.

In this paper we extend the stepwide configura tion of the two-photon process in a three-level system to the case of pulsed fields. The relaxation of the system is included in detail, with different states allowed different relaxation constants. The method of time-resolved double resonance with pulsed sources has been known to give more direct and reliable information on relaxation than the method of steady-state double resonance. Moreover, the present analysis gives analytical solutions in the intermediate-intensity regime, thus allowing application to more experimental situations.

We investigate the excitation line shape as a function of relaxation and the time dependence of the fields by deriving an equation for the position of the peaks of the Autler-Townes doublet. New pulse-width-dependent shifts are derived, studied as a function of detuning, and found to persist for even an on-resonance two-photon transition. Ef fects due to the time dependence of the amplitude 
of excitation were previously encountered in the respective responses of nonideal two- ${ }^{12}$ and threelevel systems ${ }^{13}$ to one and two near-resonance pulses. These effects involved an induced asymmetry at the long-wavelength wing of the transition.

We also study the modification of the radiative decay rates of the atom due to the influence of external fields. Owing to the nonlinear excitation, the decay rates include intensity-dependent terms which also depend on the detunings, and also on the time dependence of the field amplitudes. Such modification is neglected in the dressed-atom approach, since spontaneous emission is treated independently from excitation. ${ }^{11}$ The expressions for the decay rates may be useful to time-resolved measurements.

This paper consists of five sections. In Sec. II we solve for the two-photon probability amplitude and in Sec. III we discuss the absorption spectrum, while in Sec. IV we discuss the decay rates. Finally, we make some concluding remarks in Sec. V.

\section{PROBABILITY AMPLITUDE}

We consider a three-level atomic system with only one of the excited states optically connected to the ground state (Fig. 1). The position of this level is arbitrarily taken between the ground state and the optically forbidden higher excited state. The state of the atom $\Psi$, at any time, satisfies the equation

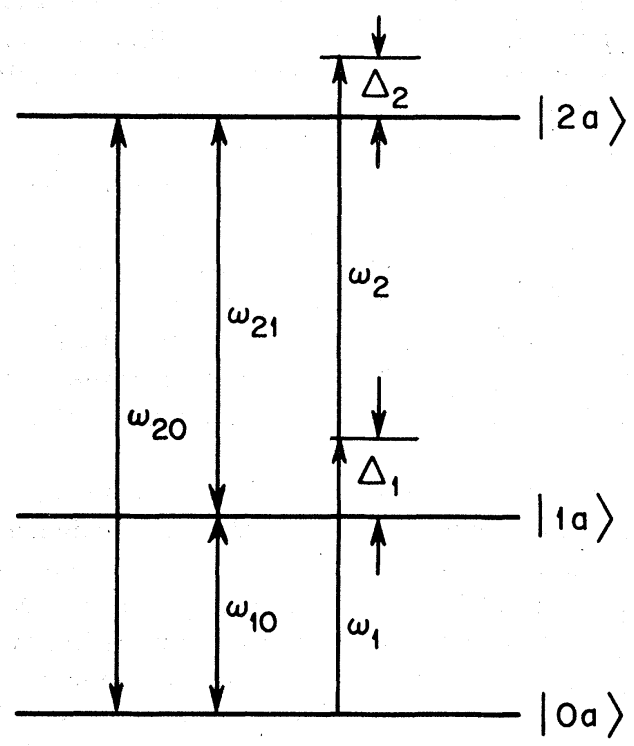

FIG. 1. Energy-level diagram of the three-level system.

$$
i \hbar \frac{\partial \psi}{\partial t}=H \psi,
$$

where the Hamiltonian $H$ is written in the electric dipole approximation as

$$
H=H_{A}-\overrightarrow{\hat{\mu}} \cdot \overrightarrow{\mathcal{E}}+H_{D},
$$

where $\overrightarrow{\hat{\mu}}$ is the atomic dipole operator, $\overrightarrow{\mathcal{E}}$ is the classical electric field vector evaluated at the center of the atom, $H_{A}$ is the Hamiltonian of the atomic system in the absence of an external field, and $H_{D}$ describes the radiative damping of the excited states. We assume that the matrix $H_{D}$ is diagonal with the elements $-\frac{1}{2} i \hbar \gamma_{1}$ and $-\frac{1}{2} i \hbar \gamma_{2}$ for the excited states and zero for the ground state.

The state of the atom may be expressed as a superposition of three orthogonal time-dependent states; that is,

$$
\begin{aligned}
\psi= & a_{0}(t)|0 a\rangle+a_{1}(t) e^{-i \omega_{10} t}|1 a\rangle \\
& +a_{2}(t) e^{-i \omega_{20} t}|2 a\rangle .
\end{aligned}
$$

Explicit forms for $a_{0}, a_{1}$, and $a_{2}$ are found by solv ing the equation of motion given the initial conditions and the electric field. We let

$$
\overrightarrow{\mathcal{E}}=2 \overrightarrow{\mathcal{E}}_{1}(t) \cos \left(\omega_{1} t\right)+2 \overrightarrow{\mathcal{E}}_{2}(t) \cos \left(\omega_{2} t\right) .
$$

We assume that $\omega_{10}$ is not near $\omega_{21}$, and therefore consider the case in which $\mathcal{E}_{1}$ and $\mathcal{E}_{2}$ induce only transitions between the levels $|0 a\rangle-|1 a\rangle$ and $|1 a\rangle-|2 a\rangle$, respectively. This approximation neglects two-photon resonant enhanced generation of mixed frequencies at $2 \omega_{1}+\omega_{2}$ and $\omega_{1}+2 \omega_{2}$, which arises when each of the field components is taken to interact with both transitions. However, for the study of local effects (photon echoes, optical nutation, adiabatic rapid passage, and optical Stark shifts), the above model is viable, especially when the intermediate state is near resonance.

In the rotating-wave approximation and for $\omega_{2}$ away from $\omega_{1}$, Eqs. (1) -(4) give the following equations for the $\Delta_{m}=0$ selection rule:

$$
\begin{aligned}
& A_{0}^{\prime}=i \mu_{01} \mathcal{E}_{1} A_{1}, \\
& A_{1}^{\prime}+i \delta_{1} A_{1}=i \mu_{10} \mathcal{E}_{1} A_{0}+i \mu_{12} \mathcal{E}_{2} A_{2}, \\
& A_{2}^{\prime}+i \delta_{2} A_{2}=i \mu_{21} \mathcal{E}_{2} A_{1},
\end{aligned}
$$

where the $\mu_{i j}$ are the elements of the dipole-operator matrix, primes denote differentiation with respect to $t$, and

$A_{0}=a_{0}, A_{1}=a_{1} \exp \left(i \Delta_{1} t\right), \quad A_{2}=a_{2} \exp \left(i \Delta_{2} t\right)$,

$\delta_{1}=-\left(\Delta_{1}+\frac{1}{2} i \gamma_{1}\right), \quad \delta_{2}=-\left(\Delta_{2}+\frac{1}{2} i \gamma_{2}\right)$,

$\Delta_{1}=\omega_{1}-\omega_{10}, \quad \Delta_{2}=\omega_{1}+\omega_{2}-\omega_{20}, \quad \Delta_{2}-\Delta_{1}=\omega_{2}-\omega_{21}$.

We determine an approximate solution to Eqs. 
(5) - (7) when the amplitudes $\mathcal{E}_{1}$ and $\mathcal{E}_{2}$ of the field are slowly varying functions of time. Eliminating $A_{0}$ and $A_{1}$ from Eqs. (5) -(7) and neglecting terms the order of $\left(\ln \mathcal{E}_{n}\right)^{\prime \prime}$ and $\left[\left(\ln \mathcal{E}_{n}\right)^{\prime}\right]^{2}$, we obtain

$$
\begin{aligned}
A_{2}^{\prime \prime}+ & {\left[i \delta-\left(\ln \mathcal{E}_{1} \mathcal{E}_{2}^{2}\right)^{\prime}\right] A_{2}^{\prime \prime} } \\
& +\left[\alpha^{2}-i \delta_{1}\left(\ln \mathcal{E}_{1} \mathcal{E}_{2}^{\prime}\right)^{\prime}-i \delta_{2}\left(\ln \mathcal{E}_{1} \mathcal{E}_{2}^{2}\right)^{\prime}\right] A_{2}^{\prime}+\left\{i \delta_{2} \mathcal{E}_{1}^{2}\right. \\
& \left.-\alpha^{2}\left(\ln \mathcal{E}_{1} \mathcal{E}_{2}\right)^{\prime}+2 \alpha \alpha^{\prime}+\mathcal{E}_{1}^{2}\left[\ln \left(\mathcal{E}_{2} / \mathcal{E}_{1}\right)^{\prime}\right]\right\} A_{2}=0,
\end{aligned}
$$

where

$$
\delta=\delta_{1}+\delta_{2}, \quad \alpha^{2}=\mathcal{E}_{1}^{2}+\mathcal{E}_{2}^{2}-\delta_{1} \delta_{2},
$$

and $\mu_{01} \mathcal{E}_{1}$ and $\mu_{12} \mathcal{E}_{2}$ are replaced by $\mathcal{E}_{1}$ and $\mathcal{E}_{2}$, as they will be in the rest of the paper. To solve Eq. (8), we first attempt to write it as the product of three first-order equations ${ }^{14}$ :

$$
\left(\frac{d}{d t}+g_{3}\right)\left(\frac{d}{d t}+g_{2}\right)\left(\frac{d}{d t}+g_{1}\right) A_{2}=0 \text {. }
$$

Expanding Eq. (10) and equating the coefficients of $d^{n} / d t^{n}$ to those in Eq. (8) results in three coupled differential equations for the $g_{i}$. The procedure we use to obtain an approximate solution for the $g_{i}$ is iterative. We write $g_{i}=G_{i}+\epsilon_{i}(i=1,2,3)$ and assume that $\left|\epsilon_{i}\right| \ll\left|G_{i}\right|$. Moreover, we assume the term $\delta_{2} \mathcal{E}_{1}^{2}$, as well as the terms which involve derivatives of the field amplitudes, to be small and therefore contribute to $\epsilon_{i}$. The lowestorder equations involve the $G_{i}$ and satisfy the following algebraic equations:

$$
G_{2}+G_{3}=i \delta, \quad G_{2} G_{3}=\alpha^{2}, \quad G_{1}=0 .
$$

Neglecting $\delta_{2} \mathcal{E}_{1}^{2}$ in these equations demands the simultaneous satisfaction of the conditions

$$
\left|\delta_{2} \mathcal{E}_{1}^{2}\right| \ll\left|\alpha^{2} \delta\right|, \quad\left|\delta_{2} \delta \mathcal{E}_{1}^{2}\right| \ll\left|\alpha^{2}\right|^{2} .
$$

These conditions are satisfied in the case of exact two-photon resonances (i.e., when $\delta_{2}=0$ ) because the neglected term vanishes identically. In this case, there is no restriction on the magnitudes of the field amplitudes; in fact Brewer and Hahn ${ }^{15}$ obtained an exact solution for constant field amplitudes. These conditions are also satisfied when amplitude $\mathcal{E}_{1}$ is weak and $\mathcal{E}_{2}$ unrestricted. Moreover, since the product $\delta_{2} \mathcal{E}_{1}^{2}$ is involved, the approximations are valid in the intermediate-intensity regime.

When the intermediate state is far from resonance $\left[\left|\delta_{1} / \mathcal{E}_{1}\right| \gg 1\right.$ and $\left.\left|\delta_{2} / \mathcal{E}_{1}\right|=O(1)\right]$, the above conditions are satisfied. However, this case can best be treated by reducing the three-level system to a two-level system. Thus one can neglect $A_{1}^{\prime}$ in comparison with $i \delta_{1} A_{1}$ in Eq. (6) and so solve for $A_{1}$ in terms of $A_{0}$ and $A_{2}$. Substituting the result into Eqs. (5) and (7), we get

$$
A_{0}^{\prime}-i\left(\mathcal{E}_{1}^{2} / \delta_{1}\right) A_{0}=i\left(\mathcal{E}_{1} \mathcal{\delta}_{2} / \delta_{1}\right) A_{2},
$$

$$
A_{2}^{\prime}+i\left[\delta_{2}-\left(\mathcal{E}_{2}^{2} / \delta_{1}\right)\right] A_{2}=i\left(\mathcal{E}_{1} \mathcal{E}_{2} / \delta_{1}\right) A_{0} .
$$

The elimination of the intermediate state results in frequency shifts and two-photon coupling. In the case of a nonresonant intermediate state, the problem of three-level systems has been reduced to that of two-level systems by a number of researchers. ${ }^{13,16-18}$

When the above conditions are satisfied in the intermediate regime we obtain the following expressions for the $g_{i}$ :

$$
\begin{aligned}
& g_{1}=i K+\left[\ln \left(\alpha^{2} / \mathcal{E}_{1} \mathcal{E}_{2}\right)\right]^{\prime}+R_{1}, \\
& g_{2}=\frac{1}{2} i(\delta-\Omega)-\frac{1}{2}\left[\ln \left(\mathcal{E}_{1} \mathcal{E}_{2}^{2} / \Omega\right)\right]^{\prime}+R_{2}, \\
& g_{3}=\frac{1}{2} i(\delta+\Omega)-\frac{1}{2}\left(\ln \mathcal{E}_{1} \mathcal{E}_{2}^{2} \Omega\right)^{\prime}+R_{3},
\end{aligned}
$$

where

$$
\begin{aligned}
K= & \frac{\delta_{2} \mathcal{E}_{1}^{2}}{\alpha^{2}}, R_{1}=\frac{\mathcal{E}_{1}^{2}}{\alpha^{2}}\left(\ln \frac{\mathcal{E}_{2}}{\mathcal{E}_{1}}\right)^{\prime}, \\
R_{2}= & -\frac{1}{2} g_{1}\left(1+\frac{\delta}{\Omega}\right)+\frac{\delta_{1}-\delta_{2}}{2 \Omega}\left(\ln \mathcal{E}_{1} \mathcal{E}_{2}^{2}\right)^{\prime} \\
& -\frac{\delta_{1}}{\Omega}\left(\ln \mathcal{E}_{1} \mathcal{E}_{2}\right)^{\prime}, \\
R_{3}= & -\frac{1}{2} g_{1}\left(1-\frac{\delta}{\Omega}\right)-\frac{\delta_{1}-\delta_{2}}{2 \Omega}\left(\ln \mathcal{E}_{1} \mathcal{E}_{2}^{2}\right)^{\prime} \\
& +\frac{\delta_{1}}{\Omega}\left(\ln \mathcal{E}_{1} \mathcal{E}_{2}\right)^{\prime}, \\
\Omega^{2}= & 4 \mathcal{E}_{1}^{2}+4 \mathcal{E}_{2}^{2}+\left(\delta_{1}-\delta_{2}\right)^{2} .
\end{aligned}
$$

Using the fact that the $g_{i}$ are slowly varying functions of $t$ to integrate Eq. (10) and substituting for the $g_{i}$ from Eqs. (13)-(15), we obtain

$$
\begin{aligned}
A_{2}= & \frac{c_{1} \mathcal{E}_{1} \mathcal{E}_{2}}{\alpha^{2}} \exp \left(-\int_{\tau}^{t}\left(i K+R_{1}\right) d t\right) \\
& +\frac{c_{2} \mathcal{E}_{1}^{1 / 2} \mathcal{E}_{2}}{\Omega^{1 / 2}\left(g_{1}-g_{2}\right)} \exp \left(-\int_{\tau}^{t}\left[\frac{1}{2} i(\delta-\Omega)+R_{2}\right] d t\right) \\
& +\frac{c_{3} \mathcal{E}_{1}^{1 / 2} \mathcal{E}_{2} \Omega^{1 / 2}}{\left(g_{1}-g_{3}\right)\left(g_{2}-g_{3}\right)} \exp \left(-\int_{\tau}^{t}\left[\frac{1}{2} i(\delta+\Omega)+R_{3}\right] d t\right),
\end{aligned}
$$

where $c_{1}, c_{2}$, and $c_{3}$ are determined from the conditions at $t=\tau$.

We note that, although Eq. (20) was derived for the case of slowly varying amplitude fields such as adiabatically switched fields, one can apply the present solution to abruptly changing fields by matching solutions having the form of Eq. (20) at the times of abrupt change. In other words, the final state before the abrupt change will serve as the initial state for the solution at the start of the change. For an atom that is in the ground state at $t=\tau$, one finds that 


$$
\begin{aligned}
c_{1} & =\frac{-\alpha_{0}^{2}}{\left(g_{10}-g_{20}\right)\left(g_{10}-g_{30}\right)}, \\
c_{2} & =\frac{\left(\mathcal{E}_{10} \Omega_{0}\right)^{1 / 2}}{g_{20}-g_{30}} \\
c_{3} & =-\left(\mathcal{E}_{10} / \Omega_{0}\right)^{1 / 2}
\end{aligned}
$$

where the subscript 0 denotes values at $t=\tau$.

\section{ABSORPTION SPECTRUM}

The first term in Eq. (20) has very slow oscillations; they are not important in the weak-field limit. The second and third terms are the Rabi oscillations of the atom. The statistical average of the excitation probability $\left|A_{2}\right|^{2}$ consists of two peaks; they are the optical analog of the AutlerTownes doublet. The doublet was first observed in the microwave region in the absorption spectrum of a weak beam probing a transition which has a common level with a saturated transition. ${ }^{6}$ Peaks for a particular set of quantities of the variables in the interaction (intermediate-state detuning, field intensity, and relaxation width) occur where the frequency of the oscillations vanishes. This gives

$$
\operatorname{Re}\left(\delta-3 K+3 i R_{1}+i \zeta\right)= \pm \operatorname{Re}(\Omega+2 i X),
$$

where

$X=-\frac{1}{2} g_{1} \frac{\delta}{\Omega}-\frac{\delta_{1}}{\Omega}\left(\ln \mathcal{E}_{1} \mathcal{E}_{2}\right)^{\prime}+\frac{\delta_{1}-\delta_{2}}{2 \Omega}\left(\ln \mathcal{E}_{1} \mathcal{E}_{2}^{2}\right)^{\prime}$,

$\zeta=\left(\ln \frac{\alpha^{2}}{\mathcal{E}_{1} \mathcal{E}_{2}}\right)^{\prime}$

We now discuss the effects of the field amplitudes and their time dependence and the relaxation of the levels on the positions of the peaks. Equation (22) is fairly complicated in the simultaneous presence of all of these effects. To get a feeling for the equation we will first consider a previously derived limiting case, for which the various limits will be taken in steps. In the absence of the time dependence of the fields - for example, in the constant-field case-Eq. (22) reduces to

$$
\operatorname{Re}(\delta-3 K)= \pm \operatorname{Re} \Omega\left(1+\delta K / \Omega^{2}\right),
$$

with $\Omega=|\Omega| e^{i \theta / 2}$ where

$$
\begin{aligned}
\sin \theta= & \left(\gamma_{2}-\gamma_{1}\right)\left(\Delta_{2}-\Delta_{1}\right)|\Omega|^{-2} \\
|\Omega|= & \left\{\left[\left(\Delta_{2}-\Delta_{1}\right)^{2}+4\left(\mathcal{E}_{1}^{2}+\mathcal{E}_{2}^{2}\right)-\frac{1}{4}\left(\gamma_{2}-\gamma_{1}\right)^{2}\right]^{2}\right. \\
& \left.+\left(\gamma_{2}-\gamma_{1}\right)^{2}\left(\Delta_{2}-\Delta_{1}\right)^{2}\right\}^{1 / 4} .
\end{aligned}
$$

When the $\mathcal{E}_{1}$ field is weak, the function $K$ becomes negligible and the peaks given by Eq. (25) are given by

$$
\operatorname{Re}(\delta \pm \Omega)=0 \text {. }
$$

In the absence of relaxation, $\delta$ and $\Omega$ become real and the position of the peaks of the Autler-Townes doublet [Eq. (28)] as a function of the detuning of the field with respect to the first transition is

$$
\Delta_{1}=-\frac{1}{2} \Delta_{s} \pm\left[\left(\frac{1}{2} \Delta_{s}\right)^{2}+\mathcal{E}_{2}^{2}\right]^{1 / 2},
$$

where $\Delta_{s}=\Delta_{2}-\Delta_{1}$ is the detuning of the second transition. The analogous case where $\Delta_{1}$ and $\Delta_{s}$ are interchanged along with $\mathcal{E}_{1}$ and $\mathcal{E}_{2}$ describes the case where $\mathcal{E}_{1}$ is strong while $\mathcal{E}_{2}$ is weak; it has been discussed in detail previously. ${ }^{9}$ Therefore we will briefly discuss Eq. (29). This result indicates that when the field is resonant with the second transition $\Delta_{s}=0$, the splitting in the case of strong $\mathcal{E}_{2}$ field is $2 \mathcal{E}_{2}$. This is what is called the modulation doubling or coherence splitting of the resonant modulation effect. Under a weak $\mathcal{E}_{2}$ field, the line shows a single peak. For offresonance excitation, where $\Delta_{s} \neq 0$ such that $\Delta_{s}$ $\gg\left|\mathcal{E}_{2}\right|$, the double-resonance signal shows two peaks, at $\Delta_{1}=0$ and $\Delta_{1}=-\Delta_{s}$. The latter peak is a two-photon resonance.

The doublet structure [Eq. (29)], encountered even when the $\mathcal{E}_{1}$ which connects the ground and first excited states is very weak and when the strong saturating amplitude $\mathcal{E}_{2}$ does not couple directly with the ground state, shows the delicacy of this process. The obvious conclusion that one should expect a single absorption component in view of the apparent involvement of a single saturated transition is incorrect. This was recently pointed out for a similar situation by Cohen-Tannoudji et al. ${ }^{11}$

We will first study the effect of the intensity of the $\mathcal{E}_{1}$ field on the doublet in the absence of the relaxation and the time variation of the amplitudes. When $\mathcal{E}_{1}$ is not very weak, the function $K$ is not negligible. A first-order correction to Eq. (29) gives

$$
\Delta_{1}=\Delta_{1}^{0}-4 K^{0},
$$

where

$$
\begin{aligned}
& \Delta_{1}^{0}=-\frac{1}{2} \Delta_{s} \pm\left[\left(\frac{1}{2} \Delta_{s}\right)^{2}+\mathcal{E}_{1}^{2}+\mathcal{E}_{2}^{2}\right]^{1 / 2}, \\
& K^{0}=-\left(\Delta_{1}^{0}+\Delta_{s}\right) \mathcal{E}_{1}^{2} /\left[\Delta_{1}^{0}\left(\Delta_{1}^{0}+\Delta_{s}\right)-4 \mathcal{E}_{1}^{2}-4 \mathcal{E}_{2}^{2}\right] .
\end{aligned}
$$

At exact two-photon resonance, where $\Delta_{1}+\Delta_{s}=0$, the function $K$ vanishes, and the restriction on the magnitudes of the $\mathcal{E}_{1}$ field amplitude is removed; the positions of the peaks are then given exactly by

$$
\Delta_{1}=-\frac{1}{2} \Delta_{s} \pm\left[\left(\frac{1}{2} \Delta_{s}\right)^{2}+\mathcal{E}_{1}^{2}+\mathcal{E}_{2}^{2}\right]^{1 / 2} .
$$


Equation (31) shows that the splitting of the doublet depends on the Rabi frequency $\left(\mathcal{E}_{1}^{2}+\mathcal{E}_{2}^{2}\right)^{1 / 2}$, since $\mathcal{E}_{1}$ is not negligible. Moreover, with moderately large $\mathcal{E}_{1}$ the function $K^{0}$ is not negligible, and therefore causes a shift in the positions of the peaks. The physical interpretation of the shift $K^{0}$ lies in the shifts encountered in multiphoton processes which involve nonrescnant intermediate states. In the weak-field case where $\mathcal{E}_{1}^{2}$ and $\mathcal{E}_{2}^{2}$ are much less than $\Delta_{1} \Delta_{2}, K^{0}$ reduces to the wellknown frequency-shift expression $\mathcal{E}_{1}^{2} / \Delta_{1}$, which is encountered in a three-level system with an intermediate state far from resonance. We note that both the absolute position and the splitting of the doublet depend on the finite Rabi frequency $\mathcal{E}_{1}$; the position of the peaks are thus asymmetric about $\Delta_{s}$, whereas they are symmetric in the cases considered above: small $\mathcal{E}_{1}$ Rabi frequency [Eq. (29)] and exact two-photon resonance [Eq. (33)].

We now consider the effect of relaxation on the splitting of the doublet and on its absolute position. We consider the case of negligible $\mathcal{E}_{1}$ frequency. - In this case the positions of the peaks depend only on the difference $\gamma_{2}-\gamma_{1}$, and therefore become independent of relaxation for equal radiative decay constants. The first-order correction in $\gamma_{2}-\gamma_{1}$ is

$\Delta_{1}=-\frac{1}{2} \Delta_{s} \pm\left\{\left(\frac{1}{2} \Delta_{s}\right)^{2}+\mathcal{E}_{2}^{2}-\left[\frac{1}{4}\left(\gamma_{2}-\gamma_{1}\right)\right]^{2}\right\}^{1 / 2}$.

Note that (34) is also valid when $\gamma_{1}$ and $\gamma_{2}$ are very different from each other, as long as both are small. The relaxation causes modulation doubling peaks to occur at $\pm\left\{\mathcal{E}_{2}^{2}-\left[\left(\frac{1}{4} \gamma_{2}-\gamma_{1}\right)\right]^{2}\right\}^{1 / 2}$ rather than at $\pm \mathcal{E}_{2}$; the peak positions around $\Delta_{s}$, however, stay symmetric.

Let us now consider some of the effects due to the time variation of the field amplitudes. Effects of this kind have recently been found in the resonant interaction of pulses with atomic systems. In the interaction of smooth pulses with two- and three-level real systems, i.e., those with finite lifetimes, it was found that the time variation of the field amplitude causes an asymmetry at the long-wavelength wing of the spectral response. ${ }^{12,13}$ It is to be noted that, in the adiabatic limit of the interaction in which the levels have long lifetimes, this asymmetry disappears. A line shift which is a cross effect of relaxation and the time variation of the field amplitude occurs in the case of double resonance. One can see that it is indeed a cross relaxation-time variation effect by taking $\gamma_{1}=\gamma_{2}$ $=0$ in Eq. (22); the quantities $\delta_{1}, \delta_{2}, \delta, \Omega, \alpha^{2}$, and $R_{1}$ become real, and thus the effect of the time variation drops out.

In order to simplify our analysis, we consider the effect of the variation of the field amplitudes on the doublet for the case of two-photon resonance, with the final state having long lifetime and the intermediate state having long but finite lifetime ( $\gamma_{1}$ is small). Note that in these limits, the solution is valid even when both $\mathcal{E}_{1}$ and $\mathcal{E}_{2}$ are arbitrarily large. In this case, the peaks of the doublet get shifted by $-\Delta_{1} \pm\left(\Delta_{1}^{2}+4 \Omega \operatorname{Im} X\right)^{1 / 2}$, where

$$
\begin{aligned}
& \Omega=\left(\frac{1}{4} \Delta_{s}^{2}+\mathcal{E}_{1}^{2}+\mathcal{E}_{2}^{2}-\frac{1}{4} \gamma_{1}^{2}\right)^{1 / 2}, \quad \Delta_{1}=-\frac{1}{2} \Delta_{s} \pm \Omega, \\
& \operatorname{Im} X=-\frac{\gamma_{1}}{2 \Omega}\left(\ln \mathcal{E}_{1} \mathcal{E}_{2}\right)^{\prime}+\frac{\gamma_{1}}{4 \Omega}\left(\ln \mathcal{E}_{1} \mathcal{E}_{2}^{2}\right)^{\prime} .
\end{aligned}
$$

For $\Delta_{1}$ large, the shift reduces to $-2 \Omega \operatorname{Im} X / \Delta_{1}$, while near $\Delta_{1} \sim 0$ the shift approaches $\pm 2(\Omega \operatorname{Im} X)^{1 / 2}$. It is worth noting that the peaks get shifted by different amounts, so that the splitting is changed; the time variations of the amplitudes [Eq. (34)] remove the symmetry of the peaks around $\frac{1}{2} \Delta_{s}$, but symmetry is retained in the limit of $\Delta_{s}$ $=0$. Figure 2 shows the variation of the shift with $\Delta_{s} / \gamma_{1}$ for field amplitudes which have $e^{-\lambda t}$ time dependence. The graph represents the relationship at the instant of time when $\left(2 \mathcal{E}_{1} / \gamma_{1}\right)^{2}+\left(2 \mathcal{E}_{2} / \gamma_{1}\right)^{2}=6$ and $\lambda / \gamma_{1}=1$

\section{DECAY RATES}

Nonlinear excitation provokes perturbation in the radiative decay of the population of the final state. The fact that the two excited states are coupled strongly indicates that the decay of the excitation will be governed by the average of the radiative decay of both levels, namely $\frac{1}{2}\left(\gamma_{1}+\gamma_{2}\right)$. In addition to this strong coupling effect, we expect intensity-dependent contributions, which are a natural consequence of high-intensity excitation. They show up in the response of a two-level sys-

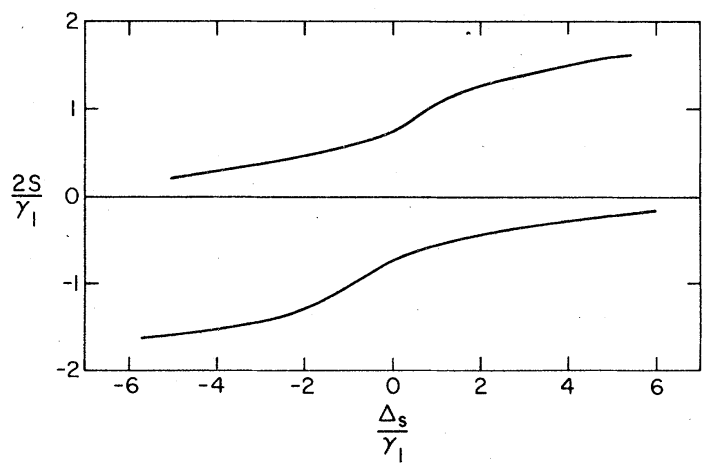

FIG. 2. Shift $S$ introduced by the time variation of the field amplitude as a function of the detuning of the field from the second transition. The shift and the detuning are in units of $\gamma_{1}$. The upper and lower curves represent the shift of the peaks at $-\frac{1}{2} \Delta_{s}+\Omega$ and $-\frac{1}{2} \Delta_{s}-\Omega$, respectively. 
tem to intense fields when the lifetimes of the levels are different, and are absent when both lifetimes are equal. In a two-photon process, the intensity-dependent terms are further complicated by the presence of the intermediate state. Moreover, the time variation of the field amplitudes in a pulsed double-resonance scheme-the case under consideration here-further contributes to the decay rate of the population.

From Eq. (20) one can easily write two decay rates associated with the population:

$$
\begin{aligned}
& q_{1}=\frac{1}{2}\left(\gamma_{1}+\gamma_{2}\right)-2 \operatorname{Re}\left(\frac{1}{2} i \Omega+R_{2}\right), \\
& q_{2}=\frac{1}{2}\left(\gamma_{1}+\gamma_{2}\right)-2 \operatorname{Re}\left(-\frac{1}{2} i \Omega+R_{3}\right)
\end{aligned}
$$

for the $\Omega, R_{1}$, and $R_{2}$ defined in Eqs. (19), (17), and (18), respectively. In the general case $q_{1}$ and $q_{2}$ are very complicated functions, but we would like to consider a special case in which the excited states have long lifetimes, namely, when $\gamma_{1}=\gamma_{2}$ $=0$. In this case $q_{1}=-q_{2}$.

$$
q_{1}=-q_{2}=-2\left(\Delta_{s} / \Omega\right)\left(\ln \mathcal{E}_{1} \mathcal{E}_{2}^{2}\right)^{\prime}+2\left(\Delta_{1} / \Omega\right)\left(\ln \mathcal{E}_{1} \mathcal{E}_{2}\right)^{\prime} .
$$

This contribution, induced by the time variation of the field amplitudes, is also a detuning-induced effect. The first term is produced by the detuning from the second transition and depends on the time variation of $\mathcal{E}_{1} \mathcal{E}_{2}^{2}$. The second term is produced by the detuning from the first transition, but is pro- portional to the time variation of $\mathcal{E}_{1} \mathcal{E}_{2}$ and of opposite sign to the first terms when $\Delta_{s}$ and $\Delta_{1}$ have the same sign. At the peaks of the AutlerTownes doublet $\Delta_{1}=-\frac{1}{2} \Delta_{s} \pm \frac{1}{2} \Omega$, and therefore

$$
q_{1}=-q_{2}= \pm\left(\ln \mathcal{E}_{1} \mathcal{E}_{2}\right)^{\prime}-\left(\Delta_{s} / \Omega\right) \ln \mathcal{E}_{1}^{3} \mathcal{E}_{2}^{5} .
$$

The first term of this equation, the lending contribution to $q_{1}$, is the rate of change of the natural $\log$ of the product of the field amplitudes, which is the sum of the spectral widths of the pulses; its magnitude is independent of the details of the detunings of the interaction. The second term, however, is proportional to $\Delta_{s}$ and $\Omega^{-1}$, and therefore makes the overall contribution detuning and intensity dependent.

\section{CONCLUSION}

The validity of the analytical treatment of pulsed double resonance presented here extends into the intermediate-intensity regime. We have examined the relaxation of the levels in detail and have derived expressions for relaxation - and pulse-shapedependent frequency shifts in the Autler-Townes doublet, and for contributions to the radiative decay rates of the levels.

\section{ACKNOWLEDGMENT}

This work is partially supported by the University of Mlinois Research Board.
${ }^{1}$ F. Schuda, C. R. Stroud, Jr., and M. Hercher, J. Phys. $\mathrm{B}$ 7, L198 (1974); H. Walther, in Proceedings of the Second Laser Spectroscopy Conference, Megev, France (Springer, Berlin, 1975); W. Harting, W. Rasmussen, R. Schieder, and H. Walther, Z. Phys. A 278, 205 (1976); F. Y. Wu, R. Grove, and S. Ezekiel, Phys. Rev. Lett. 35, 1426 (1975); R. E. Grove, F. Y. $\mathrm{Wu}$, and S. Ezekiel, Phys. Rev. 15, 227 (1977).

${ }^{2}$ B. R. Mollow, Phys. Rev. A $\underline{5}, 1522$ (1972); 8,1949 (1973).

${ }^{3}$ G. Oliver, E. Ressayre, and A. Tallet, Lett. Nuovo Cimento 2, 777 (1971); H. J. Carmichael and D. F. Walls, J. Phys. B 8, L77 (1975); 9, 1199 (1976); S. S. Hassan and R. K. Bullough, ibid. $\underline{8}$, L147 (1975); M. E. Smithers and H. S. Freedhoff, $i b i \bar{d} .8,2911$ (1975); H. J. Kimble and L. Mandel, Phys. Rev. A 13, 2123 (1976); B. Renaud, R. M. Whitley, and C. R. Stroud, Jr., J. Phys. B 9 , L19 (1976); B. R. Mollow, Phys. Rev. A 12, 1919 (1975).

${ }^{4}$ J. H. Eberly, Phys. Rev. Lett. 37, 1387 (1976); G. S. Agarwa 1, ibid . 37, 1383 (1976); P. Avan and C. CohenTannoudji, J. Phys. B 10, 155 (1977).

${ }^{5} \mathrm{~L}$. Mandel and H. J. Kimble, in Coherence and Quantum Optics, edited by L. Mandel and E. Wolf (Plenum, New York, 1978); P. Avan and C. Cohen-Tannoudji, J. Phys. B 10, 155 (1977).

${ }^{6}$ S. H. Autler and C. H. Townes, Phys, Rev. 100, 703 (1955).
${ }^{7}$ A. Schabert, R. Keil, and P. E. Toschek, Opt. Commun. 13, 265 (1975); Ph. Cahuzac and R. Vetter, Phys. Rev. A 14, 270 (1976); C. Delsart and J. C. Keller, J. Phys. B 9, 2769 (1976); J. L. Picqué and J. Pinard, ibid . 9 , L $7 \overline{7}$ (1976); J. E. Bjorkholm and P. F. Liao, Opt. Commun. 21, 132 (1977).

${ }^{8} \mathrm{H}$. R. Gray and C. R. Stroud, Jr., Opt. Commun. $\underline{25}$, 359 (1978).

${ }^{9} \mathrm{~T}$. Hänsch and P. Toschek, Z. Phys. 236, 213 (1970); $\mathrm{K}$. Shimoda, in Laser Spectroscopy of Atoms and Molecules, edited by $\mathrm{H}$. Walther (Springer, Berlin, 1976); Z. Phys. 234, 293 (1970); A. Javan, Phys. Rev. 107, 1579 (1957); B. Macke, J. Messelyn, R. Wertheimer, J. Phys. (Paris) 30, 665 (1969); B. R. Mollow, Phys. Rev. A 5, 1522 (1972); S. Feneuille and M. G. Schweighofer, J. Phys. (Paris) 36, 781 (1975).

${ }^{10}$ R. M. Whitley and C. R. Stroud, Jr., Phys. Rev. A 14, 1498 (1976).

${ }^{11} \mathrm{C}$. Cohen-Tannoudji and S. Reynaud, J. Phys. B $\underline{10}$, 345 (1977); 10, 2311 (1977).

${ }^{12}$ R. H. Lehmberg and J. Reintjes, Phys. Rev. A 12, 2574 (1975); M. H. Nayfeh, ibid. 14, 1304 (1976).

${ }^{13}$ M. H. Nayfeh and A. H. Nayfeh, in Coherence and Quantum Optics, edited by L. Mandel and E. Wolf (Plenum, New York, 1978); Phys. Rev. A 15, 1169 (1977).

${ }^{14}$ M. G. Payne and M. H. Nayfeh, Phys. Rev. A $\underline{13}, 595$ (1976).

${ }^{15}$ R. G. Brewer and E. L. Hahn, Phys. Rev. A 11,1641 
(1975).

${ }^{16}$ M. H. Nayfeh, Phys. Rev. A 14, 1304 (1976); M. H. Nayfeh and A. H. Nayfeh, ibid. 15, 1169 (1977); 18 , 1124 (1978).

${ }^{17}$ S. R. Hartmann, IEEE J. Quantum Electron QE-4, 802 (1968); E. M. Belonov and I. A. Poluektov, Zh. Eksp.
Teor. Fiz. 56, 1407 (1969) [Sov. Phys. JETP 29, 754 (1969)]; M. Takatsuji, Phys. Rev. A $\underline{4}, 808$ (1971); N. Tan-no, K. Yokoto, and H. Inaba, J. Phys. B $\underline{8}$, 339 (1975).

${ }^{18}$ D. Grischkowsky, M. M. T. Loy, and P. F. Liao, Phys. Rev. A 12, 2514 (1975). 